\title{
Subjective assessment of pain and disability in subjects with Knee-osteoarthritis undergoing low level laser therapy (LLLT)
}

\author{
Arun kumar $\mathbf{M}^{1}$, Venkatesh $D^{2, *}$ \\ ${ }^{1}$ Professor, ${ }^{2}$ Assistant Professor, Dept. of Physiology, M S Ramaiah Medical College, Bangalore,Karnataka, India \\ *Corresponding Author: \\ Email: venkatesh40@gmail.com
}

Received: $10^{\text {th }}$ January, 2018

Accepted: $25^{\text {th }}$ January, 2018

\begin{abstract}
Introduction: Osteoarthritis (OA) of knee is the causes for chronic pain and disability which makes the patient seek regular treatment. The non-pharmacological mode of management is needed for such chronic conditions as long term use of medications can have serious adverse effects. Low level laser therapy is one of the modalities of treatment which is known to reduce pain, and improve the functions.

Materials and Methods: The subjective assessment of pain and disability was done by using visual analog scale (VAS), Modified Western Ontario and McMaster Universities Arthritis Index (WOMAC) for Indian Population and Severity index of Lequesne for Knee osteoarthritis. Such scores were compared before and after Low level laser therapy for duration of 3 months.

Results: The average VAS score was $7.3 \pm 0.73$ and $4 \pm 1.76$ before and after 3 months of LLLT respectively which was statistically significant. $(\mathrm{p}=0.000)$. There was statistically significant difference in WOMAC index score and Severity index of Lequesne before and after 3 months of LLLT $(\mathrm{p}=0.000)$.

Conclusion: LLLT produces a demonstrable reduction of pain, improvement in the mobility and reduction of stiffness in subjects with senile OA of knee
\end{abstract}

Keywords: Low level laser Therapy, Cold lasers, Knee Osteoarthritis, Photobiomodulation, Pain assessment.

\section{Introduction}

Osteoarthritis of knee is the most common cause of morbidity in the elderly. As the Knee joint is involved in weight- bearing, aging causes wear and tear of cartilages in the knee joint resulting in degenerative changes. Healthy cartilage is metabolically sluggish, with slow matrix turnover and a net balance of synthesis and degradation. Cartilage in early OA or after an injury is highly active metabolically. Due to wear and tear, inflammatory markers are released which helps in the repair of cartilage to begin with. But as the age advances, the regenerating capabilities of the cartilage reduces leading to progressive damage. ${ }^{1}$

The major problem with the $\mathrm{OA}$ is restriction of movements at the joint due to pain. The joint pain of OA is typically exacerbated by activity and relieved by taking rest. The cardinal symptoms leading to the diagnosis of knee Osteoarthritis are pain which is usually of insidious in onset, reduced function at the joint, short duration of stiffness after inactivity, joint instability, buckling or giving way. The patients may also develop deformity, swelling and crepitus. The significant symptom which needs attention is persistent pain and its related psychological distress. ${ }^{2}$ Pain has long been recognized as a complex sensory and emotional experience. An individual's stable psychological characteristics (trait) and the immediate psychological context in which pain is experienced (state) both influence perception of pain. ${ }^{3}$
The subjective assessment of pain and disability in Knee OA has been done by several methods. The Nottingham Health Profile (NHP) and the Arthritis Impact Measurement Scale 2 (AIMS2), Activities of Daily Living Scale of the Knee Outcome Survey, KOOS questionnaire, Short Form 36 (SF-36) and several others. ${ }^{4}$ The most common methods of assessment are Modified Western Ontario and McMaster Universities Arthritis Index (WOMAC) for Indian Population and Severity index of Lequesne for Knee osteoarthritis. ${ }^{5,6}$

There are several pharmacological and nonpharmacological options available for the managements of Knee OA. Osteoarthritis symptoms, primarily pain, may be reduced by medications like non-steroidal antiinflammatory drugs (NSAIDs). Education, physical therapy with exercise and weight loss are the mainstays in the non-pharmacological management of OA along with the promotion of general health. ${ }^{7}$ The Low level Laser therapy is the most common nonpharmacological modality of management of several disease conditions. LLLT is known to effective in pain management and promote wound healing. ${ }^{8,9}$

In this study, the subjective assessment of pain and disability in subjects with Knee-osteoarthritis undergoing low level laser therapy (LLLT) done.

\section{Materials and Methods}

The study was done in Ramaiah Medical College and teaching hospitals. Sixteen symptomatic subjects 
with clinically diagnosed knee osteoarthritis in the age group of 45-75 years were included in the study. A radiological criterion was Kellgren-Lawrence (KL) system grading II, III and IV was adopted. Subjects with KL grade I, infective arthritis, history of vascular diseases in the lower limb, photosensitivity, and patient on steroid therapy were excluded from the study. Procedure was explained and informed consent was obtained from the all subjects participating in the study.

Low level laser therapy (LLLT) was administered using a laser device with probe giving power output of maximum 10 milliwatts, with a wavelength of $830 \mathrm{~nm}$. LLLT was given with the laser probe around the 6 points around the surface of the affected knee joint. ${ }^{10}$ The dosage at each point was $1.5 \mathrm{~J}$ for the duration of 60 seconds. Thus the total dosage administered per session was 12 J. Dosage was and duration (in seconds) of LLLT was calculated for each point according the established guidelines with a total of 6 points around the knee Joint.

Modified Western Ontario and McMaster Universities Arthritis Index (WOMAC) for Indian Population and Severity index of Lequesne for Knee osteoarthritis was used for subjective assessment of symptoms in all the subjects.

Visual analog scale (VAS) was used to assess pain perception for every session of laser therapy. Weekly average of VAS was taken to access the pain perception in the individual.

Modified WOMAC (CRD- Pune version) consists of 27 questions in four different categories like Pain (5 questions), Stiffness, ${ }^{2}$ Difficulty range (17 questions) and optional 3 questions. Each question has five responses starting from none, mild, moderate, severe and extreme. The questionnaires were administered before the commencement and after the completion of therapy.

Severity index of Lequesne (algofunctional index) is the questionnaire which is specific for knee OA. It is used for assessing the prognosis. There are 3 sections for this index: ${ }^{1}$ pain or discomfort, ${ }^{2}$ maximum distance walked and ${ }^{3}$ activities of daily living. Scoring is given for items under these sections. Index of severity was obtained by adding the scores for all parameters. Interpretation is done based on the minimum points for each section is 0 , maximum points for each section are 8 , minimum index score is 0 , and maximum index score is 24 . The questionnaires were administered before the commencement and after the completion of therapy.

The baseline parameters of the participants were mentioned as Mean \pm SD in the study. The scores obtained by administration of questionnaire before and after the completion of intervention were compared by using paired $t$ test. $\mathrm{p}<0.05$ was considered to be statiscally significant.

\section{Results}

Total of 25 subjects participated in the study out of which only 18 completed the study. 7 patients dropped out before 1 month of the study. The average age of the participants who participated in the study was 60 years. Other baseline parameters are mentioned in the table 1. The average BMI of the participants were $27 \mathrm{~kg} / \mathrm{m}^{2}$. The average duration of low level laser therapy was 60 sec per point.

The scores of Visual analog scale, WOMAC index and Severity index of Lequesne for Knee osteoarthritis are represented in the table 2. The average VAS was 7.3 \pm 0.73 and $4 \pm 1.76$ before and after 3 months of LLLT. The difference was statistical significant $(\mathrm{p}=0.000)$. Visual analog score on weekly basis showed that there is a slight increase in the pain during first week and followed by progressive decrease thereafter. This is depicted in graph 1 .

WOMAC index score before and after 3 months of LLLT was $57.1 \pm 7.33$ and $44.65 \pm 7.27$ respectively with statistically significant difference $(\mathrm{p}=0.000)$. Severity index of Lequesne exhibited statistically significant difference before and after 3 months of LLLT. The values were $16.15 \pm 1.23$ and $13.85 \pm 1.60$ $(\mathrm{p}=0.000)$.

Table 1: Total number of participants with Baseline parameters in the study

\begin{tabular}{|l|l|c|}
\hline S. No & \multicolumn{1}{|c|}{$(\mathbf{n = 1 8})$} & Number \\
\hline 1 & Gender & \\
& Male & 13 \\
& Female & 5 \\
\hline 2 & K L Grading on Radiography & \\
& Grade II & 13 \\
& Grade III & 4 \\
& Grade IV & 1 \\
\hline 3 & Affected Knee R/L & \\
& Right side & 12 \\
& Left side & 6 \\
\hline
\end{tabular}


Table 2: The subjective score if different pain indices in the participants

\begin{tabular}{|l|c|c|c|}
\hline \multicolumn{1}{|c|}{ Index } & Before & After & p value \\
\hline Visual Analog scale & $7.3 \pm 0.73$ & $4 \pm 1.76$ & $0.000^{*}$ \\
\hline WOMAC index & $57.1 \pm 7.33$ & $44.65 \pm 7.27$ & $0.000^{*}$ \\
\hline Severity index of Lequesne & $16.15 \pm 1.23$ & $13.85 \pm 1.60$ & $0.000^{*}$ \\
\hline
\end{tabular}

$* \mathrm{p}<0.05$ is considered as statistically significant

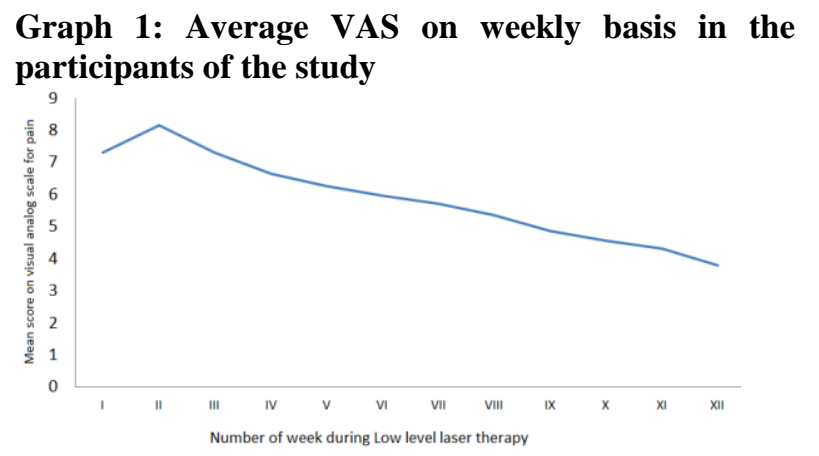

\section{Discussion}

The low level lasers therapy is known to reduce to the pain and inflammation in several tissues. This is due to the photo-biomodulation, the effect of light seen on the living tissues. The LLLT done using the wavelength of $830 \mathrm{~nm}$ has better penetration into the deeper tissues, even reaching the cartilage. ${ }^{11}$ The energy is absorbed by the deeper tissues and this energy is used for the repairing of the damaged cells, hence helping the regeneration of the damaged cartilage. In this process, there is reduction in the pain producing substances and also pro-inflammatory markers. Thus pain is reduced and inflammation is controlled leading to favourable environment for the commencement of repair process. Similar findings are presented in the study by Lucas et al 2000 according to which laser is known to heal the wounds rapidly. ${ }^{9}$

In a study done by Hamblin 2013, the low level lasers can be used in the management of osteoarthritis of joints. ${ }^{12}$ In a study by Ho Jong et al 2012, administrating LLLT around the specific joint areas caused a marked pain relief and produced other benefits like reducing inflammation, improving functions of the joint. ${ }^{13}$

In all the subjects who participated in the study, there was reduction in the pain over 3 month's duration and also there was improvement in the functions. However, there was an increase in the pain during first week of treatment. The reason could be that the inflammatory process has been accelerated in the first few days due to the low level laser therapy followed by rapid progression towards the reparative environment in the tissues. Low level laser therapy basically prevents the release of prostaglandins and other inflammatory mediators thus, reducing pain perception. ${ }^{14}$ Other mechanism of action could be LLLT acts on sodium potassium pump, alters the nerve excitation and conduction thus decreasing the pain perception..$^{15}$ LLLT may stimulate and activate the production of endogenous endorphins which act on the mu receptors and prevent the action of substance $\mathrm{P}$ thus reducing pain. ${ }^{16}$

"Pain" is an "Unpleasant sensory and emotional experience associated with actual or potential tissue damage. It is subjective in nature, as the pattern varies with every individual. ${ }^{17}$ Chronic pain in kneeosteoarthritis is a state where pain outlasts the normal time of healing and thus serves no biologic purpose rather causing persistent unpleasant sensation to the sufferer. So managing such chronic pain needs long lasting regular treatment until the reparative process in triggered in the affected joint areas. LLLT seems to be an effective mode in such conditions which not only reduces pain but also helps in repairing and restoring the damaged tissues. ${ }^{18}$

It can be concluded that the preliminary observations in the limited number of subjects with LLLT point towards a demonstrable improvement in the mobility, reduction of pain and stiffness in senile OA of knee. However the conclusively inference can be drawn by compiling the results from substantial number of subjects treated with LLLT. It will be ideal for the patients who are motivated to undergo nonpharmacological management of $\mathrm{OA}$ for better compliance and long term benefits.

\section{References}

1. Creamer P1.Osteoarthritis pain and its treatment.Curr Opin Rheumatol. 2000;12(5):450-5.

2. Hunter DJ, McDougall JJ, Keefe FJ. The symptoms of $\mathrm{OA}$ and the genesis of pain. Rheum Dis Clin North Am. 2008 Aug; 34(3):623-43.

3. Kane RL, Bershadsky B, Lin WC, Rockwood T, Wood $\mathrm{K}$, Kane RL, et al. Efforts to standardize the reporting of pain. Journal of Clinical Epidemiology. 2002;55(2):105110.

4. Rodriguez-Merchan EC. Knee instruments and rating scales designed to measure outcomes. J Orthop Traumatol. 2012 Mar;13(1):1-6.

5. Gandek B. Measurement properties of the Western Ontario and McMaster Universities Osteoarthritis Index: a systematic review. Arthritis Care Res (Hoboken). 2015 Feb;67(2):216-29.

6. GeroldStucki, Oliver Sangha, Susanne Stucki, Beat A. Michel, Alan Tyndall. Walter Dick and Robert Theiler. Comparison of the WOMAC (Western Ontario and McMaster Universities) osteoarthritis index and a selfreport format of the self-administered LequesneAlgofunctional index in patients with knee and hip osteoarthritis. Osteoarthritis and Cartilage 1998;6:79-86.

7. Anandacoomarasamy A, March L. Current Evidence for Osteoarthritis Treatments. Ther Adv Musculoskelet Dis. $2010 \mathrm{Feb} ; 2(1): 17-28$. 
8. Ying-Ying Huang, Aaron C.-H. Chen et al, Biphasic Response of Low Level Laser Therapy, Dose-Response. Ann Biomed Eng. 2012 February; 40(2): 516-533. doi:10.1007/s10439-011-0454-7.

9. C. Lucas, R.W. Stanborough et al, Efficacy of Low-Level Laser Therapy on Wound Healing in Human Subjects: A Systematic Review, Lasers Med Sci 2000,15:84-93.

10. Be' la Hegedus, La' szlo' Viharos, Miha' lyGervain, and $\mathrm{Ma}^{\prime}$ rtaGa' lfi. The Effect of low-Level Laser in Knee Osteoarthritis: A Double-Blind, Randomized, PlaceboControlled Trial. Photomedicine and Laser Surgery 2009;27, (4);577-84.

11. Hoon Chung, Tianhong Dai, Sulbha K. Sharma. The Nuts and Bolts of Low-level Laser (Light) Therapy. Ann Biomed Eng. 2012;40(2):516-33.

12. Hamblin MR. Can osteoarthritis be treated with light? Arthritis Res Ther. 2013 Oct 29; 15(5):120. doi: 10.1186/ar4354.

13. Ho Jang, Hyunju Lee. Meta-Analysis of Pain Relief Effects by Laser Irradiation on Joint Areas. Photomedicine and Laser Surgery 2012;30(8):407-17.

14. Honmura, A., M. Yanase, J. Obata and E. Haruki. Therapeutic effect of Ga-Al-As diode laser irradiation on experimentally induced inflammation in rats. Lasers Surg. Med., 1992;12:441-49.

15. Chow, R., P. Armati, E.L. Laakso, J.M. Bjordal and G.D. Baxter. Inhibitory effects of laser irradiation on peripheral mammalian nerves and relevance to analgesic effects: A systematic review. Photomed. Laser Surg., 2011;29:35681.

16. Laakso, E.L. and P.J. Cabot. Nociceptive scores and endorphin-containing cells reduced by Low-Level Laser Therapy (LLLT) in inflamed paws of Wistar rat. Photomed. Laser Surg.2005;23:32-5.

17. T. Tripathi, G. Ganesh and P. Rai. Pain Pathways and Effect of Low Level Laser Therapy on Pain During Fixed Orthodontic Treatment-an Overview. European Journal of Dentistry and Medicine 2016;8:1-5.

18. Kingsley JD, Demchak T, Mathis R. Low-level laser therapy as a treatment for chronic pain. Front Physiol [Internet]. 2014 Aug 19;5. 